Z. Klin. Chen. Klin. Biochem.

10. Jg. 1972 ,S. $270-274$

\title{
Über den Lipidstoffwechsel im Magnesium-Mangẹl. Untersuchungen an einem Arteriosklerose-Modell
}

\author{
Von J. Schmalbeck, W. R. Willems, F. Dorn und Th. Günther \\ Pbysiologisch-Chemisches Institut und Zentralinstitut für Biochemie und Biophysik der \\ Freien Universität Berlin
}

(Eingegangen am 12. November 1971)

Bei der durch Mg-arme Ernährung erzeugten Arteriosklerose von Ratten waren im Serum die Konzentrationen von Cholesterin, Triglyceriden und Phosphatiden sowie das Spektrum der verschiedenen Lipoproteine gegenüber Kontrolltieren nicht verändert. Der Gehalt des Serum an freien Fettsäuren war erhöht. Der Serumproteingehalt war besonders durch eine Abnahme der Albumine und $\gamma$-Globuline verringert.

Diese Ergebnisse sind unabhängig vom Gehalt der Mg-armen Nahrung an mehrfach ungesättigten Fettsäuren.

Der Gehalt von Cholesterin, Triglyceriden und Phosphatiden in den Aorten und Lebern wurde durch die Mg-arme Ernährung nicht beeinflußt. Eine Störung im Lipidstoffwechsel konnte als Ursache der durch Mg-Mangel bedingten Arteriosklerose ausgeschlossen werden.

\section{Lipid metabolism during a dietary magnesium deficiency. \\ Studies on artherosclerosis induced by magnesium deficiency}

Artherosclerosis was induced in rats by a diet deficient in magnesium. Compared with the non-artherosclerotic controls, the serum concentrations of cholesterol, triglycerides and phospholipids and the spectrum of various lipoproteins were unchanged in the artherosclerotic animals. The concentration of free fatty acids was increased. The serum protein concentration showed a decrease, which was due mainly to decreases in the albumin and $\gamma$-globulin fractions. These results were not influenced by changes in the levels of polyunsaturated fatty acids in the $\mathrm{Mg}$-deficient diet. The concentrations of cholesterol, triglycerides and phospholipids in the aortas and livers of $\mathrm{Mg}$-deficient tats were the same as in the controls. These results show that Mg-deficiency artheroscletosis cannot be correlated with a disturbance in lipid metabolism.

Mg-Mangel-Ennährung führt im Tierversuch zur Verkalkung der Arterienwände. Das elektronenmikroskopische Bild dieser Veränderung ist nicht identisch mit der Arteriosklerose nach Cholesterinverfütterung (1). Ob eine Hypercholesterinämie an der Entstehung der Arteriosklerose des Mg-Mangels beteiligt ist, ist unklar. Von Arkawa und Mitarbeitern (2) wurde eine Ethöhung der Serum-Cholesterin-Konzentration nach Mgarmer Ernährung beschrieben, nach anderen Autoren (3) ist der Cholesterinspiegel jedoch unverändert.

Hypercholesterinämie und Arteriosklerose nach Cholesterinteicher Ennährung konnten von einigen Untersuchern $(4,5,6)$ durch $\mathrm{Mg}$-Gaben vermindert werden, von anderen (7) jedoch nicht. Die Verkalkung der Gefäßwand im Mg-Mangel wird durch hohen Cholesteringehalt des Futters verstärkt (7) und die Serum-MgKonzentration zusätzlich gesenkt $(8,9)$.

Die möglichen biochemischen Zusammenhänge zwischen Cholesterin- und Mg-Konzentration sowie das Verhalten und die Rolle der anderen Lipidfraktionen bei der Gefäßverkalkung im Mg-Mangel sind nicht bekannt.

Zur Klärung dieser Beziehungen untersuchten wir die Lipide in Serum, Leber und Aorta nach $\mathrm{Mg}$-armer Ernährung.

\section{Material und Methoden}

Versuchstiere

Die Versuche wurden an $120-150 \mathrm{~g}$ schweren, männlichen Wistarratten durchgefühtt. Je 15-20 Tiere wurden nach folgendem Schema über eine Versuchsdauer von 5 Wochen ernährt:

Gruppe 1 exhielt die bereits beschriebene künstliche $\mathrm{Mg}$-arme Versuchsdiät (10). Ihr $\mathrm{Mg}$-Gehalt betrug $1,5 \mathrm{mMol} / \mathrm{kg}$.

Gruppe 2 erhielt die künstliche $\mathrm{Mg}$-reiche Diät (Mg-Gehalt: $16 \mathrm{mMol} / \mathrm{kg}$ ).

Gruppe 3 wurde, um einen möglichen Einfluß verminderter Nahrungsaufnahme berücksichtigen zu können, mit der $\mathrm{Mg}$ reichen künstlichen Diät in reduzierter Menge so gefüttert, daß ihr Gewichtszuwachs dem der $\mathrm{Mg}$-arm ernährten Tiere entsprach. Gruppe 4 erhielt Normalfutter (Altromin R)

Alle Versuchsgruppen mit Ausnahme der Gruppe 3 erhielten das Futter ad libitum. Allen Versuchstieren stand uneingeschränkt destilliertes Wasser zur Verfügung.

Gewinnung des Versuchsmaterials

Am Versuchsende wurde nach 12-stdg. Fasten den Tieren durch Herzpunktion in leichter Äthernarkose Blut entnommen, welches bis zur Abtrennung des Serums und der sofortigen Weiterverarbeitung bei $0^{\circ}$ gehalten wurde.

Einzelne Leberlappen und die Aorta thoracalis wurden nach der Entnahme in flüssigem $\mathrm{N}_{2}$ eingefroren und gefriergetrocknet. Die Aorta wurde sorgfältig aus ihrer Umgebung freipräpariert, in eiskalter KREBs-RINGer-Lösung unter einer Präparationslupe von 
anhaftendem Binde- und Fettgewebe befreit und die Präparate histologisch kontrolliert. Bei der Bestimmung des Triglyceridgehaltes trat eine starke Streuung der Meßwerte auf, was wahrscheinlich auf einen unterschiedlichen Gehalt an anhaftendem Fettgewebe zurückzuführen ist. $\mathrm{Da}$ der gemessene Triglyceridgehalt niemals $3 \%$ des Trockengewichtes überschritt, sind die mitgeteilten Werte der anderen Lipide in der Aorta, die auf das Trockengewicht bezogen wurden, hierdurch jedoch nicht wesentlich verfälscht.

\section{Analysen im Serum}

Die Bestimmung der Serum-Mg-Konzentration erfolgte durch Atomabsorptionsspektrophotometrie nach Enteiveißung mit 10proz. Trichloressigsäure, die $0,1 \% \mathrm{La}$ (als $\mathrm{LaCl}_{3}$ ) enthielt. Der Serum-Eiweißgehalt wurde nach LowRY und Mitarbeitern (11) gemessen. Das Gesamt-Cholesterin im Serum bestimmten wir mit einer modifizierten LrebermanN-BurChARD-Reaktion (12), die Phosphatide nach den Angaben von ZilvisRsmrt und Mitarbeitern (13). Der Serum-Triglyceridgehalt wurde nach Verseifung durch enzymatische Bestimmung des freigesetzten Glycerins ermittelt $(14,15)$. Zur Bestimmung der freien Fettsäuren verwendeten wir die titrimetrische Methode von Dols und Mitarbeitern (16). Die elektrophoretische Auftrennung der Proteine erfolgte auf Celluloseacetatfolien, die Proteine wurden mit Ponceau $S$ und die Lipoproteine mit Olrot $O$ angefärbt (17). Die Auswertung wurde densitometrisch durchgeführt.

\section{Gewebs-Analysen}

Zur Lipidanalyse wurden die Gewcbsproben nach der Methode von Folch und Mitarbeitern (18) extrahiert. Die Triglyceride wurden durch Messung des freigesetzten Glycerins nach Verseifung bestimmt $(14,15)$, die Gesamtphosphatide nach BARTLETT (19). Die Phosphatide der Aorta wurden dünnschichtchromatographisch aufgetrennt, die einzelnen Fraktionen eluiert und der Phosphorgehalt der Eluate gemessen (20). Das Cholesterin bestimmten wir mit einer Mikromodifikation der Methode von AbELL und Mitarbeitern (21). Der eingeengte Lipidextrakt wurde mit etwa $0,5 \mathrm{~N}$ äthanol. $\mathrm{KOH}$ verseift und mit Petroläther ( $\mathrm{Kp}$ $50-70^{\circ}$ ) und Wasser ausgeschüttelt. Die Farbreaktion im Petroläther-Auszug wurde jedoch nicht mit dem LIEBERMANN-BurCHARDReagenz, sondern in Gegenwart von $\mathrm{Fe}[\mathrm{III}]-$ Perchlorat, Essigsäureäthylester und konz. Schwefelsäure durchgeführt (22).

\section{Ergebnisse}

Im Laufe des Experimentes entwickelten alle Mg-arm ernährten Tiere die typischen $\mathrm{Mg}$-Mangel-Symptome (6): vorübergehende Hyperämie der Akren, Haut- und Schleimhautnekrosen, z. T. auch Tetanien. Der Gewichtszuwachs war signifikant erniedrigt. Die Serum$\mathrm{Mg}-$ Konzentration war am Versuchsende von 0,9 $\mathrm{mMol} / 1$ auf $0,49 \mathrm{mMol} / \mathrm{l}$, der Serum-Eiweißgehalt um etwa $15 \%$ abgesunken, wobei hauptsächlich das Albumin und die $\gamma$-Globuline betroffen waren (Tab. 1). Aus diesen Befunden folgt, daß sich die Mg-arm ernährten Tiere am Ende des Versuches im Mg-Mangelzustand befanden.

\section{Serumlipide}

Die Analysenwerte sind in Tabelle 2 zusammengefaßt. Der Cholesterin- und Phosphatidgehalt des Serums war bei allen mit der künstlichen Diät ernährten Tieren gleich, aber etwas höher als bei den mit Altromin gefütterten Ratten. Dieser Effekt könnte auf dem höheren Fettgehalt der Versuchsdiät (10 Gewichts $\%$ entsprechend $21 \mathrm{Kal} . \%$ ) gegenüber Altromin (5 Gewichts\% entsprechend 12,5 Kal.\%) beruhen (23). Bei den $\mathrm{Mg}$ Mangeltieren und bei den „Hungertieren“ war die Serum-Triglyceridkonzentration gering, aber nicht signifikant erhöht. Der Gehalt an freien Fettsäuren im Serum lag im Mg-Mangel ungefähr $60 \%$ und bei reduzierter Nahrungsaufnahme etwa $25 \%$ über den Werten der Vergleichsgruppen. $\mathrm{Da}$ die Lipide im Serum als Lipoproteine transportiert werden, welche eine hohe Syntheserate in der Leber aufweisen (24), andererseits aber die Proteinsynthese, insbesondere die Albumin-Synthese, im Mg-Mangel gestört ist (25), untersuchten wir auch das Lipoproteinspektrum im Serum. Der erhöhten

Tab. 1

Gewichtszuwachs, Serum-Mg-Konzentration, Gesamteiweiß-, Albumin- und Globulingehalt im Serum von Ratten nach 5-wöchiger Ernährung mit Mg-armer, Mg-reicher oder mengenmäßig reduzierter, Mg-reicher (= „Hungertiere") Diät sowie mit Altromin. Angegeben sind die Mittelwerte und ihr mittlerer Fehler

\begin{tabular}{|c|c|c|c|c|c|c|c|c|c|}
\hline & $\begin{array}{c}\text { Gewichts- } \\
\text { zuwachs } \\
\text { während } 5 \\
\text { Wochen } \\
{[\mathrm{g}]}\end{array}$ & $\begin{array}{c}M g \\
{[\mathrm{mMol} / 1]}\end{array}$ & $\begin{array}{c}\text { Protein } \\
{[\mathrm{g} / 1]}\end{array}$ & $\begin{array}{l}\text { Albumin* } \\
\text { [g/l] }\end{array}$ & $\begin{array}{c}\alpha_{1} \\
{[\mathrm{~g} / \mathrm{l}]}\end{array}$ & $\begin{array}{c}a_{2} \\
{[\mathrm{~g} / \mathrm{l}]}\end{array}$ & $\begin{array}{c}\text { lobuline* } \\
\alpha_{3} \\
{[\mathrm{~g} / 1]}\end{array}$ & $\begin{array}{c}\beta \\
{[\mathrm{g} / \mathrm{l}]}\end{array}$ & $\begin{array}{c}\gamma \\
{[\mathrm{g} / I]}\end{array}$ \\
\hline $\begin{array}{l}\text { Gruppe 1 } \\
M g \text {-arm } \\
(\mathrm{n}=20)\end{array}$ & $\begin{array}{c}38,6 \\
\pm 2,7 \\
\text { (a) }\end{array}$ & $\begin{array}{r}0,49 \\
\pm 0,02 \\
\text { (a) }\end{array}$ & $\begin{array}{l}60,2 \\
\pm 0,7 \\
(a)\end{array}$ & $\begin{array}{l}29,2 \\
\pm 0,4 \\
(\text { a) }\end{array}$ & $\begin{array}{r}10,7 \\
\pm 0,4\end{array}$ & $\begin{array}{r}4,2 \\
\pm 0,3\end{array}$ & $\begin{array}{r}3,8 \\
: \pm 0,4\end{array}$ & $\begin{array}{r}8,3 \\
\pm 0,2\end{array}$ & $\begin{array}{l}4,0 \\
\pm 0,3 \\
(a)\end{array}$ \\
\hline $\begin{array}{l}\text { Gruppe } 2 \\
M g-r e i c h \\
(n=19)\end{array}$ & $\begin{array}{l}94,5 \\
\pm 2,8 \\
\text { (b) }\end{array}$ & $\begin{array}{l}0,87 \\
\pm 0,05 \\
\text { (b) }\end{array}$ & $\begin{array}{l}69,0 \\
\pm 0,8 \\
\text { (b) }\end{array}$ & $\begin{array}{l}34,0 \\
\pm 0,4 \\
\text { (b) }\end{array}$ & $\begin{array}{r}10,6 \\
\pm 0,8\end{array}$ & $\begin{array}{r}3,9 \\
\pm 0,3\end{array}$ & $\begin{array}{r}3,1 \\
\pm 0,3\end{array}$ & $\begin{array}{r}9,9 \\
\pm 0,4\end{array}$ & $\begin{array}{c}7,6 \\
\pm 0,4 \\
\text { (b) }\end{array}$ \\
\hline $\begin{array}{c}\text { Gruppe } 3 \\
\text { "Hungertiere" } \\
(n=15)\end{array}$ & $\begin{array}{r}33,1 \\
\pm 3,2\end{array}$ & $\begin{array}{r}0,89 \\
\pm 0,05 \\
\text { (b) }\end{array}$ & $\begin{array}{l}68,7 \\
\pm 1,4 \\
\text { (b) }\end{array}$ & $\begin{array}{l}32,4 \\
\pm 0,4 \\
\text { (b) }\end{array}$ & $\begin{array}{r}12,4 \\
\pm 0,3\end{array}$ & $\begin{array}{r}3,8 \\
\pm 0,2\end{array}$ & $\begin{array}{r}3,0 \\
\pm 0,2\end{array}$ & $\begin{array}{r}8,0 \\
\pm 0,3\end{array}$ & $\begin{array}{l}9,1 \\
\pm 0,2 \\
\text { (b) }\end{array}$ \\
\hline \multirow[t]{2}{*}{$\begin{array}{l}\text { Gruppe } 4 \\
\text { Altromin } \\
(n=20)\end{array}$} & & $\begin{array}{r}1,05 \\
\pm 0,02 \\
\text { (b) }\end{array}$ & $\begin{array}{c}69,7 \\
\pm 1,2 \\
\text { (b) }\end{array}$ & $\begin{array}{l}33,4 \\
\pm 0,6 \\
\text { (b) }\end{array}$ & $\begin{array}{r}9,9 \\
\pm 0,6\end{array}$ & $\begin{array}{r}3,8 \\
\pm 0,3\end{array}$ & $\begin{array}{r}3,5 \\
\pm 0,2\end{array}$ & $\begin{array}{r}9,2 \\
\pm 0,5\end{array}$ & $\begin{array}{r}9,9 \\
\pm 0,7 \\
\text { (b) }\end{array}$ \\
\hline & $\begin{array}{c}\text { (a) (b) } \\
p<0,001\end{array}$ & $\begin{array}{c}\text { (a) (b) } \\
\mathrm{p}<0,001\end{array}$ & $\begin{array}{c}\text { (a) (b) } \\
\mathrm{p}<0,01\end{array}$ & $\begin{array}{c}\text { (a) (b) } \\
\mathrm{p}<0,01\end{array}$ & & & & & $\begin{array}{c}\text { (a) (b) } \\
\mathrm{p}<0,01\end{array}$ \\
\hline
\end{tabular}

* Zufällige Stichprobe von je 10 Tieren. 
Tab. 2

Lipidgehalt und Lipoproteinspektrum des Serums von Ratten nach 5-wöchiger Ernährung mit Mg-armer, Mg-reicher oder mengenmäßig reduzierter, Mg-reicher (= "Hungertiere") Diät sowie mit Altromin. Angegeben sind die Mittelwerte und ihr mittlerer Fehler

\begin{tabular}{|c|c|c|c|c|c|c|c|}
\hline & \multirow{2}{*}{$\begin{array}{c}\text { Cholesterin } \\
{[\mathrm{g} / \mathrm{l}]}\end{array}$} & \multirow{2}{*}{$\begin{array}{l}\text { Triglyceride } \\
{[\mathrm{g} / \mathrm{l}]}\end{array}$} & \multirow{2}{*}{$\begin{array}{c}\text { Phosphatide } \\
{[\mathrm{g} / \mathrm{l}]}\end{array}$} & \multirow{2}{*}{$\begin{array}{c}\text { Freie } \\
\text { Fettsäuren } \\
{[\mathrm{mMol} / 1]}\end{array}$} & \multicolumn{3}{|c|}{ Lipoproteinspektrum* } \\
\hline & & & & & AID & [rel. $\%$ ] & $\beta$ \\
\hline $\begin{array}{c}\text { Gruppe } 1 \\
\text { Mg-arm } \\
(\mathrm{n}=20)\end{array}$ & $\begin{array}{l}0,66 \\
\pm 0,03 \\
\text { (a) }\end{array}$ & $\begin{array}{r}0,66 \\
\pm 0,05\end{array}$ & $\begin{array}{r}1,05 \\
\pm 0,02\end{array}$ & $\begin{array}{c}0,39 \\
\pm 0,013 \\
\text { (a) }\end{array}$ & $\begin{array}{r}5,0 \\
\pm 0,7\end{array}$ & $\begin{array}{r}71,9 \\
\pm 2,2\end{array}$ & $\begin{array}{r}22,1 \\
\pm 1,8\end{array}$ \\
\hline $\begin{array}{l}\text { Gruppe } 2 \\
\text { Mg-reich } \\
(n=19)\end{array}$ & $\begin{array}{c}0,64 \\
\pm 0,02 \\
\text { (a) }\end{array}$ & $\begin{array}{r}0,56 \\
\pm 0,05\end{array}$ & $\begin{array}{r}0,98 \\
\pm 0,05\end{array}$ & $\begin{array}{c}0,25 \\
\pm 0,018 \\
\quad \text { (b) }\end{array}$ & $\begin{array}{r}3,3 \\
\pm 0,5\end{array}$ & $\begin{array}{r}76,5 \\
\pm 2,2\end{array}$ & $\begin{array}{r}20,4 \\
\pm 2,0\end{array}$ \\
\hline $\begin{array}{c}\text { Gruppe } 3 \\
\text { „Hungertiere“ } \\
(n=15)\end{array}$ & $\begin{array}{c}0,66 \\
\pm 0,02 \\
\text { (a) }\end{array}$ & $\begin{array}{r}0,67 \\
\pm 0,04\end{array}$ & $\begin{array}{r}1,20 \\
\pm 0,05\end{array}$ & $\begin{array}{c}0,31 \\
\pm 0,023 \\
\text { (b) }\end{array}$ & $\begin{array}{r}4,9 \\
\pm 0,5\end{array}$ & $\begin{array}{r}74,5 \\
\pm 0,8\end{array}$ & $\begin{array}{r}20,6 \\
\pm 0,8\end{array}$ \\
\hline $\begin{array}{l}\text { Gruppe } 4 \\
\text { Altromin } \\
(n=20)\end{array}$ & $\begin{array}{l}0,55 \\
\pm 0,02 \\
\text { (b) } \\
\text { (a) }(\mathrm{b}) \\
\mathrm{p}<0,05\end{array}$ & $\begin{array}{r}0,56 \\
\pm 0,03\end{array}$ & $\begin{array}{r}0,79 \\
\pm 0,03\end{array}$ & $\begin{array}{c}0,24 \\
\pm 0,013 \\
\text { (b) } \\
\text { (a) (b) } \\
p \pm 0,05\end{array}$ & $\begin{array}{r}3,9 \\
\pm 0,7\end{array}$ & $\begin{array}{r}70,3 \\
\pm 3,5 \\
\end{array}$ & $\begin{array}{r}24,7 \\
\pm 2,9\end{array}$ \\
\hline
\end{tabular}

* Zufällige Stichprobe von je 10 Tieren.

1) Nach dem Vorschlag von SEIDEL wurde Albumin den Lipoproteinen zugerechnet (39).

Konzentration an freien Fettsäuren entsprechend fand sich bei den Mg-Mangelratten und bei-den „Hungertieren" eine vermehrte Anfärbbarkeit der Albuminfraktion auf Lipide. Die $\alpha$ - und $\beta$-Lipoproteine zeigten keine Unterschiede zwischen den verschiedenen Versuchsgruppen.

Ein unterschiedlicher Gehalt der Mg-armen Versuchsdiät an mehrfach ungesättigten Fettsäuren hatte keinen Einfluß auf den Gehalt des Serums an Cholesterin, Triglyceriden und freien Fettsäuren, wie wir in einem Versuch feststellten, in dem die Versuchsdiät 10\%(w/w) Olivenöl statt $10 \%(w / w)$ Maisöl enthielt.

\section{Leberlipide}

Da die Leber im Lipidstoffwechsel eine zentrale Stellung einnimmt, bestimmten wir den Gehalt dieses Organes an verschiedenen Lipiden. Es ließen sich keine Unter-
Tab. 3

Lipidgehalt (in $\mathrm{mg} / \mathrm{g}$ Trockengewicht) der Lebern von Ratten, die 5 Wochen mit Mg-armer, Mg-reicher oder mengenmäßig reduzierter, Mg-reicher (= „Hungertiere") sowie mit Altromin ernährt wurden. Angegeben sind Mittelwerte und ihr mittlerer Fehler

\begin{tabular}{cccc}
\hline & Cholesterin & Triglyceride & Phosphatide \\
\hline Gruppe 1 & 7,83 & 31,2 & 118 \\
Mg-arm & $0,38 \pm$ & $\pm 1,5$ & $\pm 6,0$ \\
(n=20) & & & \\
Gruppe 2 & 7,14 & 35,9 & 111 \\
Mg-reich & $+0,22$ & $+2,7$ & 5,7 \\
(n=19) & & & \\
Gruppe 3 & 7,06 & 26,6 & 115 \\
,Hungertiere، & $\pm 0,41$ & $\pm 2,0$ & $\pm 4,6$ \\
(n=15) & & & 117 \\
Gruppe 4 & 6,95 & 20,3 & $\pm 3,5$ \\
Altromin & $\pm 0,15$ & $\pm 1,2$ & \\
(n=20) & & & \\
\hline
\end{tabular}

Tab. 4

Lipidgehalt (in mg/g Trockengewicht) der Aorten von Ratten, die 5 Wochen mit Mg-armer, Mg-reicher oder mengenmäßig reduzierter, Mg-reicher ( = „Hungertiere“) Diät sowie mit Altromin gefüttert wurden. Angegeben sind die Mittelwerte und ihr mittlerer Fehler

\begin{tabular}{|c|c|c|c|c|c|}
\hline & Cholesterin & $\begin{array}{l}\text { Gesamt-Phos- } \\
\text { phatide }\end{array}$ & Lecithin* & Sphingomyelin* & Kephaline* \\
\hline $\begin{array}{c}\text { Gruppe } 1 \\
M g \text {-arm } \\
(n=20)\end{array}$ & $\begin{array}{r}4,17 \\
\pm 0,10\end{array}$ & $\begin{array}{c}14,1 \\
\pm 0,52\end{array}$ & $\begin{array}{r}5,8 \\
\pm 0,3\end{array}$ & $\begin{array}{r}4,5 \\
\pm 0,2\end{array}$ & $\begin{array}{r}2,8 \\
\pm 0,4\end{array}$ \\
\hline $\begin{array}{l}\text { Gruppe } 2 \\
\text { Mg-reich } \\
(\mathrm{n}=19)\end{array}$ & $\begin{array}{r}4,66 \\
\pm 0,16\end{array}$ & $\begin{array}{c}14,5 \\
\pm 0,52\end{array}$ & $\begin{array}{r}6,1 \\
\pm 0,3\end{array}$ & $\begin{array}{r}4,1 \\
\pm 0,3\end{array}$ & $\begin{array}{r}2,8 \\
\pm 0,2\end{array}$ \\
\hline $\begin{array}{c}\text { Gruppe } 3 \\
\text { "Hungertiere“ } \\
(n=15)\end{array}$ & $\begin{array}{r}4,32 \\
\pm 0,07\end{array}$ & $\begin{array}{c}14,0 \\
\pm 0,57\end{array}$ & $\begin{array}{r}6,2 \\
\pm 0,3\end{array}$ & $\begin{array}{r}4,1 \\
\pm 0,4\end{array}$ & $\begin{array}{r}2,8 \\
\pm 0,2\end{array}$ \\
\hline $\begin{array}{l}\text { Gruppe } 4 \\
\text { Altromin } \\
(n=20)\end{array}$ & $\begin{array}{r}4,61 \\
\pm 0,10\end{array}$ & $\begin{array}{c}15,0 \\
\pm 0,51 \\
?\end{array}$ & $\begin{array}{r}6,9 \\
\pm 0,3\end{array}$ & $\begin{array}{r}4,5 \\
\pm 0,1\end{array}$ & $\begin{array}{r}2,7 \\
\pm 0,1\end{array}$ \\
\hline
\end{tabular}

* Zufällige Stichprobe von je 5 Tieren. 
schiede im Cholesterin-, Triglycerid- und Phosphatidgehalt nachweisen, mit Ausnahme eines niedrigeren Triglyceridgehaltes der Lebern der mit Altromin ernährten Ratten, der sich wahrscheinlich durch den unterschiedlichen Fett- und Kohlenhydratgehalt der Diäten erklären läßt (23) (Tab. 3).

\section{Aortenlipide}

Bei der spontanen Arteriosklerose des Menschen und verschiedener Versuchstiere steigt der Gehalt von Lipiden, besonders des Cholesterins, in den Arterien an (26-29). Bei der Arteriosklerose der Ratte im MgMangel konnten wir bei den Versuchsgruppen keine signifikanten Änderungen im Gehalt an Cholesterin, Gesamtphosphatiden, Kephalinen, Lecithin und Sphingomyelin in den Aorten feststellen (Tab. 4).

\section{Diskussion}

Im Mg-Mangel tritt bei Ratten keine Änderung in der Serum-Cholesterin-Konzentration auf, womit wir die von Vitale und Mitarbeitern (3) an jungen Hunden gemachte Beobachtung bestätigen. $\mathrm{Da}$ das Lipoproteinspektrum ebenfalls unverändert ist, kann sich der physiko-chemische Zustand des Serum-Cholesterin nicht wesentlich geändert haben. Änderungen des Serum-Cholesterin und der Lipoproteine lassen sich daher als Ursachen der Arteriosklerose des Mg-Mangels ausschließen.

Damit übereinstimmend waren auch der CholesterinGehalt und der Cholesterin-turnover in der Aorta Mgarm ernährter Ratten unverändert. Daraus folgt, daß die geringe Vermehrung der Triglyceride und der freien Fettsäuren im Serum - beide Substanzen werden als mitwirkende Faktoren bei der Ausbildung einer Arteriosklerose diskutiert $(30-32)$ - keinen Einfluß auf die Permeation von ${ }^{3} \mathrm{H}-$ Cholesterin in die Aorta hatten (33).

Bei spontan verkalkenden Gefäßen kommt es mit Ausbildung der Gefäßwandveränderungen zu einer geringen Zunahme des Gesamtphosphatidgehaltes, wobei der Sphingomyelinanteil weitaus am stärksten zunimmt
$(27,29)$. Auch diese Alteration im Lipidstoffwechsel der Arterien ließ sich im Mg-Mangel nicht nachweisen. Die Lipidanalysen der Aorten bestätigen somit die elektronenmikroskopischen Ergebnisse (1), wonach sich im Mg-Mangel eine Arteriosklerose ohne sichtbare Anhäufung von Lipiden ausbildet. Sie kommt wahrscheinlich durch quantitative (und vielleicht auch qualitative) Veränderungen des Elastins zustande $(1,34)$. Vielleicht kann sich auch beim Menschen eine Arterioșklerose ohne Änderung des Lipidstoffwechsels infolge einer Störung des Elastinstoffwechsels, z. B. bei länger andauernder Hypomagnesiämie, entwickeln.

Im Mg-Mangel kommt es zu einer Erhöhung der freien Fettsäuren im Serum. Dieser Befund läßt sich nicht allein durch verminderte Nahrungsaufnahme und einen daraus resultierenden Hungerzustand der $\mathrm{Mg}$-arm ernährten Tiere erklären, denn die Konzentration an freien Fettsäuren war bei den „Hungertieren“ (Gruppe 3) deutlich niedriger. Die Vermehrung der freien Fettsäuren läßt sich wahrscheinlich auf eine erhöhte Lipolyse zurückführen, die wir im braunen Fettgewebe Mg-arm ernährter Ratten nachweisen konnten (35). Ihre Ursachen werden an anderer Stelle diskutiert (35). Wie sich das weiße Fettgewebe im Mg-Mangel verhält, ist unklar (35).

Eine erhöhte Konzentration von freien Fettsäuren im Serum führt in der Leber zu einer verstärkten Fettsynthese und vermehrten Sekretion von Lipoproteinen in das Serum $(36,37)$. Dadurch ließe sich dessen geringfügig vermehrter Triglyceridgehalt bei $\mathrm{Mg}$-Mangeltieren (und „Hungertieren ") erklären. Demnach scheint die Synthese des Proteinanteils der Lipoproteine in der Leber nicht gehemmt, denn bei ihrer Hemmung hätte eine Zunahme des Triglyceridgehaltes der Leber und, wegen ihrer kurzen Halbwertszeit (38), eine starke $\mathrm{Ab}$ nahme der Triglyceride im Serum eintreten müssen. Offenbar ist in der Leber im Mg-Mangel nur die an membrangebundenen Ribosomen ablaufende Proteinsynthese, z. B. des Albumins (25), vermindert.

Frau L. Richter und Frau A. Thiele danken wir für ihre gewissenhafte Mitarbeit.

\section{Literatur}

1. Merker, H. J. und TH. GüNTher, diese Z. 8, 374 (1970). 2. Arkawa, J. K. und A. P. David, Ann. N. Y. Acad. Sci. 162, 744 (1969). - 3. Vitale, J. J., E. E. Helterstern, M. NakaMURA und B. Lown, Circulation Res. 9, 387 (1961). - 4. NAKAmura, M., J. J. Vitale, D. M. Hegsted und E. E. Hellerstein, J. Nutrit. 71, 347 (1960). - 5. Mullick, D. N. und K. B. KAKKaR, Indian J. Med. Res. 51, 742 (1963). - 6. WALSER, M., Erg. Physiol. 59, 185 (1967). - 7.. Nakamura, M., S. Torir, M. Hiramatsu, J. Hirano, A. Sumiyoshi und K. Tanaka, J. Atheroscler. Res. 5, 145 (1965). - 8. Hellerstein, E. E., M. Nakamura, D. M. Hegsted und J. J. Vitale, J. Nutrit. 71, 339 (1960). - 9. Bhattacharyya, N. V. und D. N. Mullick, Ann. Biochem. exper. Med. 23, 515 (1963). - 10. Güntrier, Th., diese Z. 8, 65 (1970). 11. Lowry, O. H., N. J. Rosebrovgh, A. L. Farr und R. J. Randall, J. biol. Chemistry 193, 265 (1951). - 12. Watson, D., Clin. chim. Acta Amsterdam 5, 637 (1960). - 13. Zilversmit,
D. B. und A. K. Davis, J. Laborat. Clin. Med. S. Louis 35, 155 (1950). - 14. Eggstein, M. und F. H. Kreutz, Klin. Wschr. 44, 262 (1966). - 15. Schmidd, F. H. und K. v. DAFL, diese Z. 6 , 156 (1968). - 16. Dole, V. P. und H. Meinertz, J. biol. Chemistry 235, 2595 (1960). - 17. Klemens, U. H. und J. SchmalBECK, diese Z. 7, 540 (1969). - 18. FoLCH, J., M. LEES und G. H. Sloane-Stanley, J. biol. Chomistry 226, 497 (1957). - 19. Bartlett, G. R., J. biol. Chemistry 234, 466 (1959). - 20 Skipski, V. P., R. F. Peterson und M. Barclay, Biochem. J. 90, 374 (1964). - 21. Abelc, L. L., B. B. Levy, B. B. Brodie und F. E. Kendall, J. biol. Chemistry 195, 357 (1952). - 22. Wybenga, D. R., V. J. Pileggi, P. H. Dirstine und J. Di Giorgio, Clin. Chem. New York 16, 980 (1970). - 23. Angelico, R., L. Moretta, G. Improta und P. L. IAlongo, Nutr. Metabol. 12, 179 (1970). - 24. MARSH, J. B., in "Structural and functional aspects of lipoproteins in living systems", ed. by E. Tria and 
A. M. Scanu, p. 447, Acad. Press, London-New York (1969). 25. Schwartz, R., N. A. Woodcock, J. D. Blakely, F. L. Wang und E. A. Khatrallah, J. Nutrit. 100, 123 (1970). - 26. BötrCHER, C. J. F., S. P. WOOdFORd, C. TER HAAR ROMENY-WACHter, E. Boelsma van Houte und C. M. van Gent, Lancet 1960/I, 1378. - 27. BötTCher, C. J. F. und S. P. WOODFORd, Fed. Proc. Suppl. 11, 21, 15 (1962). - 28. Smith, E. B., J. Atheroscler. Res. 5, 224 (1965). - 29. SMIrH, E. B., R. S. SLATER und P. K. ChU, J. Atheroscler. Res. 8, 399 (1968). - 30. KIEF, H., Naturwissenschaften 57; 384 (1970). - 31. WELBORN, T. A., D. J. A. Jenkins, G. N. Cumpston, D. H. Curnow, D. V. Goff, C. J. Johnstone, N. S. Stenhouse und M. Sumarers, in "A therosclerosis, Proc. of the Second Intern. Symp.“, ed. by R. J.
JONES, p. 369, Springer-Verlag, Berlin-Heidelberg-New York (1970). - 32. Carlson, I. A., 1. c. (31), p. 516. - 33. SchmalBECK, J., F. Dorn, H. J. Merker und Th. Günther, diese $Z$. 1.0, 275 (1972). - 34. BRITTON, W. M. und E. L. R. Stoxstad, J. Nutrit. 100, 1501 (1970). - 35. GüNTher, Th., J. ScharalBECK, F. Dorn und H. J. MrRker, diese Z. in Vorbereitung. 36. Carlson, L. A. und S. O. Lirjedahr, Acta med. Scand. 178, 81 (1965). - 37. Basso, L. V. und R. J. HAVEL, Clin. Res. 15, 137 (1967). - 38. Girtin, D., D. G. Cornwele, D. Nakasato, J. L. Oncley, W. L. Hughes, jr. und C. A. Janeway, J. Clin. Invest. 36, 172 (1957). - 39. SerDei,' D., PlasmalipoproteineFunktion und Charakterisierung: in „Fettstoffwechselstörungen“" (G. Schettler ed.), Georg Thieme Verlag, Stuttgart (1971) S. 24.

Prof. Dr. Th. Günther 1000 Berlin 33 Arnimallee 22 